$$
\begin{aligned}
& \text { UNITU THEP 94-3 } \\
& \text { sw } 9411
\end{aligned}
$$

\title{
The Bethe-Salpeter equation for mesons as quark - anti-quark bound states in a soliton background ${ }^{\dagger}$
}

\author{
H. Weigel ${ }^{\ddagger}$ and R. Alkofer \\ Institute of Theoretical Physics \#, Tübingen University, \\ Auf der Morgenstelle 14, D-72076 Tübingen, Germany
}

March 4, 1994

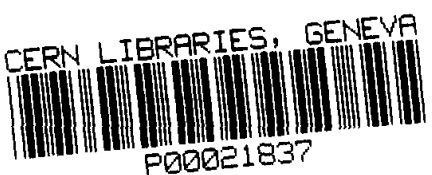

\section{Abstract}

A program is provided which solves the Bethe-Salpeter equation for meson fields which are bound in the background field of a localized soliton configuration. To this end the energy and the properly normalized wave-function of the bound state are obtained by identifying the vanishing eigenvalue of the bilocal Bethe-Salpeter kernel. Special care is taken to minimize the computational time needed to obtain the bound state energy.

† Supported by the Deutsche Forschungsgemeinschaft (DFG) under contract Re 856/2-1.

‡ Supported by a Habilitanden-scholarship of the DFG.

\# E-mail to: weigel@mailserv.zdv.uni-tuebingen.de

UNITU-THEP-3/1994

Submitted to: Computer Physics Communications. 


\section{PROGRAM SUMMARY}

Title of the program: bpwave

Catalogue number:

Program obtainable from: CPC Program Library, Queen's University of Belfast, N. Ireland (see application form in this issue).

Computer: UNIX WORKSTATIONS: SUN-SPARC, DEC-5000, HP-9000.

Installation: Institute of Theoretical Physics, Tübingen University.

Operating system: UNIX.

Programming language used: FORTRAN 77.

High speed storage required:

SUN: $6.85 \mathrm{MB}$ data and stack segments, $5.08 \mathrm{MB}$ real memory;

DEC: $6.90 \mathrm{MB}$ virtual size, $5.22 \mathrm{MB}$ real memory;

HP: $3.44 \mathrm{MB}$ virtual size.

Peripherals used: standard input, standard output, disk.

Keywords: Nambu-Jona-Lasinio model, chiral soliton, P-wave kaon fluctuation, BetheSalpeter equation, bilocal kernel, bound state, strangeness, hyperons.

Nature of the physical problem

The program calculates the bound state energy of a $\mathrm{P}$-wave kaon fluctuation in the background field of the chiral soliton in the Nambu-Jona-Lasinio model[1]. Also the bound state wave-function is evaluated and normalized to carry unit strangeness charge[2].

\section{Method of solution}

The bilocal kernel of the Bethe-Salpeter equation is discretized and extended to an eigenvalue problem. The bound state energy is identified as the one which leads to at least one vanishing eigenvalue. The corresponding eigenvector is identified as the bound state wave-function.

Restriction of the complexity of the problem

The program is confined to strange $\mathrm{P}-$-wave fluctuations in the soliton background. Generalizations are possible without changing the structure of the program.

\section{Typical running time}

The running time strongly depends on the size of the momentum space basis as well as the number of lattice points. Furthermore the desired accuracy of the solution plays an important role. Typical parameters approximately yield $250 \mathrm{~min}$ (SUN), 310min (DEC), 140min (HP) cpu-time. 
Unusual features of the program

The program needs two input files ("chi.in" and "VEC.DAT") created by the numerical routine "chisol" described in ref.[3].

References

[1] H. Weigel, H. Reinhardt and R. Alkofer, Phys. Lett. B313 (1993) 377.

[2] H. Weigel, R. Alkofer and H. Reinhardt, "Hyperons in the bound state approach to the Nambu-Jona-Lasinio chiral soliton", Tübingen preprint Oct. 93, HEP-PH/9310309.

[3] R. Alkofer and H. Weigel, "Self-consistent solution to a fermion determinant with space dependent fields", UNITU-THEP-1/1994. Submitted to Computer Physics Communications.

\section{LONG WRITE UP}

\section{The physical problem}

The main purpose of this program is to construct the kaon bound state in the background field of the self-consistent chiral soliton[1] of the Nambu-Jona-Lasinio (NJL) model[2]. This provides a natural description of the low-lying hyperons. Such calculations have been initiated by Callan and Klebanov[3] in the context of the Skyrme model[4]. The numerical determination of the self-consistent chiral soliton of the NJL model has been reported in ref. [5] on which the current numerical routine is also based.

As the starting point of the physical problem we assume the bosonized version[6] of the three flavor ( $u p$, down, strange) NJL model with scalar and pseudoscalar degrees of freedom. The corresponding action, $\mathcal{A}_{N J L}$ splits into a mesonic part

$$
\mathcal{A}_{m}=\int d^{4} x\left(-\frac{1}{4 G_{\mathrm{NJL}}} \operatorname{tr}\left(M^{\dagger} M-\hat{m}^{0}\left(M+M^{\dagger}\right)+\left(\hat{m}^{0}\right)^{2}\right)\right) .
$$

and a fermion determinant

$$
\mathcal{A}_{F}=\operatorname{Tr} \log (i \not D)=\operatorname{Tr} \log \left(i \not \partial-\left(P_{R} M+P_{L} M^{\dagger}\right)\right)
$$


such that $\mathcal{A}_{N J L}=\mathcal{A}_{F}+\mathcal{A}_{m}$. Here $G_{\mathrm{NJL}}$ and $\hat{m}^{0}=\operatorname{diag}\left(m_{u}^{0}, m_{d}^{0}, m_{s}^{0}\right)$ denote the NJL coupling constant and the current quark mass matrix, respectively. Isospin symmetry $m_{u}^{0}=m_{d}^{0}=: m^{0}$ is assumed while SU(3) symmetry is violated $m^{0} \neq m_{s}^{0}$. $P_{R, L}$ are projectors on right- and lefthanded quark spinors. $M=S+i P$ is a $3 \times 3$ complex matrix field and represents scalar $(S)$ and pseudoscalar $(P)$ mesons. Since the NJL model is not renormalizable a regularization prescription has to be imposed. We adopt Schwinger's proper time description[7]. Then one more parameter, the cut-off $\Lambda$, is introduced. $S$ acquires a space-time independent, non-vanishing vacuum expectation value $\langle S\rangle=\operatorname{diag}\left(m_{u}, m_{d}, m_{s}\right)=: \operatorname{diag}\left(m, m, m_{s}\right)$. The constituent quark masses $\left(m, m_{s}\right)$ are the solutions to the Schwinger-Dyson equations. In the proper time regularization scheme they read[1]

$$
m_{i}=m_{i}^{0}+m_{i}^{3} \frac{N_{C} G_{N J L}}{2 \pi^{2}} \Gamma\left(-1,\left(\frac{m_{i}}{\Lambda}\right)^{2}\right)
$$

where $N_{C}=3$ denotes the number of colors. In the meson sector the experimental values for the pion and kaon masses $\left(m_{\pi}=135 \mathrm{MeV}\right.$ and $\left.m_{K}=495 \mathrm{MeV}\right)$ are employed to further fix the parameters of the model. These physical masses $\left(m_{\text {phys }}\right)$ are incorporated by demanding the corresponding propagators to acquire a pole, or equivalently the inverse propagators to possess vanishing eigenvalues

$$
\operatorname{det}\left(D_{i j, k l}^{-1}\left(q^{2}=m_{\mathrm{phys}}^{2}\right)\right)=0
$$

which represent the Bethe-Salpeter equations for free mesons. In the NJL model the inverse propagators are given by

$$
D_{i j, k l}^{-1}\left(q^{2}\right)=\left(-\frac{\left(m_{i}^{0}+m_{j}^{0}\right)\left(m_{i}+m_{j}\right)}{2 G_{\mathrm{NJL}}}-\Pi_{i j}\left(q^{2}\right)\right) \delta_{i l} \delta_{k j} .
$$

The first term on the RHS stems from $\mathcal{A}_{m}$ while the fermion determinant yields the polarization tensor[8]

$$
\Pi_{i j}\left(q^{2}\right)=-2 q^{2} f_{i j}^{2}\left(q^{2}\right)+2\left(m_{i}-m_{j}\right)^{2} f_{i j}^{2}\left(q^{2}\right)-\frac{1}{2}\left(m_{i}^{2}-m_{j}^{2}\right)\left(\frac{\langle\bar{q} q\rangle_{i}}{m_{i}}-\frac{\langle\bar{q} q\rangle_{j}}{m_{j}}\right)
$$

wherein

$$
\langle\bar{q} q\rangle_{i}=-m_{i}^{3} \frac{N_{c}}{4 \pi^{2}} \Gamma\left(-1, M_{i}^{2} / \Lambda^{2}\right)
$$

are the quark condensates. The physical decay constants may be extracted from

$$
f_{i j}^{2}\left(q^{2}\right)=\frac{1}{4}\left(m_{i}+m_{j}\right)^{2} \frac{N_{c}}{4 \pi^{2}} \int_{0}^{1} d x \Gamma\left(0,\left[(1-x) m_{i}^{2}+x m_{j}^{2}-x(1-x) q^{2}\right] / \Lambda^{2}\right) .
$$

via

$$
f_{\pi}^{2}=f_{u d}^{2}\left(m_{\pi}^{2}\right), \quad f_{K}^{2}=f_{u s}^{2}\left(m_{K}^{2}\right) .
$$

Substituting finally $f_{\pi}=93 \mathrm{MeV}$ leaves only one parameter undetermined which we choose to be the non-strange constituent quark mass $m . f_{K}$ is left as a prediction. In the pion channel (4) yields $G_{\mathrm{NJL}}=m^{0} \mathrm{~m} / \mathrm{m}_{\pi}^{2} f_{\pi}^{2}$. This relation will frequently be used to express the the ratio $G_{\mathrm{NJL}} / m^{0}$ in terms of input quantities. 
The aim is to investigate space-time dependent pseudoscalar meson fluctuations $\eta_{a}(x)$ in the background field of the static chiral soliton $\xi_{0}(\mathbf{r})$. For the latter the hedgehog shape is assumed $\xi_{0}(\boldsymbol{r})=\exp \left(\frac{i}{2} \boldsymbol{\tau} \cdot \hat{\mathbf{r}} \Theta(r)\right)$. The numerical determination of chiral angle $\Theta(r)$ is presented in ref.[5]. A convenient parametrization of the meson fields is given by[9]

$$
M=\xi_{0} \xi_{f}\langle S\rangle \xi_{f} \xi_{0}
$$

which describes the fluctuations via ${ }^{1} \xi_{f}=\exp \left(i \eta_{a}(x) \lambda_{a} / 2\right)$. Next, the action is expanded up to second order in the fluctuations $\eta_{a}$. The leading term - being independent of $\eta_{a}-$ renders the static energy functional of the chiral angle $\Theta(r)[10]$. The self-consistent solution prevents the term linear in $\eta_{a}$ from being non-zero[9]. Thus the non-trivial part of the action is quadratic in $\eta_{a}$. In order to extract this piece the functional trace in eqn.(2) has to be performed. The full calculation is described in refs. $[9,11]$. Thus we will only indicate the key steps and provide the necessary definitions. First, the temporal part of the trace is calculated analytically by Fourier analyzing the fluctuations ${ }^{2}$

$$
\eta_{a}(\mathbf{r}, t)=\int_{-\infty}^{+\infty} \frac{d \omega}{2 \pi} \tilde{\eta}_{a}(\mathbf{r}, \omega) \mathrm{e}^{-i \omega t}
$$

In the second step the trace over all remaining degrees of freedom is evaluated by introducing eigenstates and -functions

$$
h_{(0)}|\mu\rangle=\epsilon_{\mu}|\mu\rangle
$$

of the static one-particle Dirac Hamiltonian

$$
h_{(0)}(\mathbf{r})=\boldsymbol{\alpha} \cdot \boldsymbol{p}+\left(\begin{array}{cc}
m \exp \left(i \gamma_{5} \boldsymbol{\tau} \cdot \hat{\mathbf{r}} \Theta(r)\right) & 0 \\
0 & m_{s}
\end{array}\right)
$$

Hereby the eigenstates with strangeness \pm 1 are free Dirac spinors whereas those corresponding to the light quarks are functionals of the soliton. The Hamiltonian $h_{(0)}$ has the appreciated feature that it commutes with the grand spin operator

$$
G=J+\frac{\tau}{2}=l+\frac{\sigma}{2}+\frac{\tau}{2}
$$

which is the sum of the total spin $\boldsymbol{J}$ and the isospin $\boldsymbol{\tau} / 2$. The spin itself is decomposed into orbital angular momentum $\boldsymbol{l}$ and intrinsic spin $\sigma / 2$. Then the eigenstates (12) are also eigenstates of $\boldsymbol{G}^{2}=G(G+1)$ and $G_{3}=M$. For the free strange quarks we have $\boldsymbol{G}=\boldsymbol{J}$. However, it is more useful to label the strange quarks by their orbital angular momentum $l$ and consider $j=l \pm 1 / 2$ as a function of it. The eigenstates are constructed as linear combinations of the eigenstates $|\mu\rangle^{0}$ of the free Hamiltonian $(\Theta=0)$. Furthermore, certain boundary conditions have to be imposed in order to discretize the momenta of $|\mu\rangle^{0}$. Two sets of boundary conditions have been discussed in appendix $A$ of ref.[8]. Here we use the one which demands the upper component of the Dirac spinors to vanish at the boundary of a spherical cavity with radius $D$. The continuum limit which eventually should be considered corresponds to $D \rightarrow \infty$.

\footnotetext{
${ }^{1} \lambda_{a}$ are the Gell-Mann matrices.

${ }^{2}$ For this calculation it is necessary to continue forth and back from Minkowski to Euclidean space.
} 
For the problem at hand, the description of hyperons, it is sufficient to only consider kaonic fluctuations which decouple from the pions at second order. We therefore write

$$
\sum_{a=1}^{8} \check{\eta}_{a}(\mathbf{r}, \omega) \lambda_{a}=\left(\begin{array}{cc}
0 & \check{K}(\mathbf{r}, \omega) \\
\dot{K}^{\dagger}(\mathbf{r},-\omega) & 0
\end{array}\right)
$$

wherein $\check{K}(\mathbf{r}, \omega)$ is a two-component isospinor. The kaon bound state emerges as a $\mathrm{P}-$ wave fluctuation. Making furthermore use of isospin invariance one can reduce the radial dependence to just one unknown function $\eta_{\omega}(r)$ :

$$
\dot{K}(\mathbf{r}, \omega)=\hat{\mathbf{r}} \cdot \tau \eta_{\omega}(r) \Omega(\omega) \quad \text { with } \quad \Omega(\omega)=\left(\begin{array}{c}
a_{1}(\omega) \\
a_{2}(\omega)
\end{array}\right),
$$

where $a_{i}(\omega)(i=1,2)$ are Fourier amplitudes which in the process of quantization become annihilation (and creation) operators.

The part of the action which is a quadratic functional of the fluctuation $\eta_{\omega}(r), \mathcal{A}^{(2)}\left[\eta_{\omega}\right]$ receives contributions form three pieces: (i) the valence part, $\mathcal{A}_{\text {val }}$ which originates from the explicit occupation of the valence quark level; (ii) the vacuum contribution to the fermion determinant $\mathcal{A}_{\text {vac }}$; (iii) the purely mesonic part $\mathcal{A}_{m}$. The resulting action functional can be cast into a form which contains local $\Phi^{(1)}(r)$ and bilocal $\Phi^{(2)}\left(\omega ; r, r^{\prime}\right)$ kernels

$$
\begin{aligned}
\mathcal{A}^{(2)}\left[\eta_{\omega}\right]= & \int_{-\infty}^{+\infty} \frac{d \omega}{2 \pi}\left(a_{1}^{\dagger}(\omega) a_{1}(\omega)+a_{2}^{\dagger}(\omega) a_{2}(\omega)\right) \\
& \times\left\{\int d r^{2} \int d r^{\prime} r^{\prime 2} \Phi^{(2)}\left(\omega: r, r^{\prime}\right) \eta_{\omega}(r) \eta_{\omega}\left(r^{\prime}\right)+\int d r r^{2} \Phi^{(1)}(r) \eta_{\omega}(r) \eta_{\omega}(r)\right\} .
\end{aligned}
$$

For the presentation of these kernels it is convenient to introduce the unitary, self-adjoint transformation matrix ${ }^{3}$

$$
u_{0}(\mathbf{r})=\beta\left(\sin \frac{\Theta}{2}-i \gamma_{5} \hat{\mathbf{r}} \cdot \tau \cos \frac{\Theta}{2}\right)
$$

and define transformed wave-functions

$$
\xi_{\mu}(\mathbf{r})=u_{0}(\mathbf{r}) \psi_{\mu}(\mathbf{r})
$$

$\psi_{\mu}(\mathbf{r})$ denotes the coordinate space representation of the state $|\mu\rangle$. The transformation (19) only affects non-strange spinors.

The purely mesonic action, $\mathcal{A}_{m}$, only contributes to $\Phi^{(1)}(r)$ which does not depend on the frequency $\omega$

$$
\begin{gathered}
\Phi^{(1)}(r)=-\frac{\pi}{2} m_{\pi}^{2} f_{\pi}^{2}\left(1+\frac{m_{s}}{m}\right)\left(\cos \Theta+\frac{m_{s}^{0}}{m^{0}}\right)-\frac{N_{C}}{4} \eta_{\text {val }}\left(m+m_{s}\right) \int \frac{d \Omega}{4 \pi} \tilde{\psi}_{\text {val }}^{\dagger}(\mathbf{r}) \beta \tilde{\psi}_{\text {val }}(\mathbf{r})(20) \\
-\frac{N_{C}}{4}\left(m+m_{s}\right) \int_{1 / \Lambda^{2}}^{\infty} \frac{d s}{\sqrt{4 \pi s}} \int \frac{d \Omega}{4 \pi}\left\{\sum_{\mu=n s} \epsilon_{\mu} \epsilon^{-s \epsilon_{\mu}^{2}} \tilde{\psi}_{\mu}^{\dagger}(\mathbf{r}) \beta \dot{\psi}_{\mu}(\mathbf{r})+\sum_{\rho=s} \epsilon_{\rho} \epsilon^{-s \epsilon_{\rho}^{2}} \psi_{\rho}^{\dagger}(\mathbf{r}) \beta \psi_{\rho}(\mathbf{r})\right\} .
\end{gathered}
$$

The integral $\int(d \Omega / 4 \pi)$ indicates that the average with regard to the internal degrees of freedom have been taken. These traces involve matrix elements which are listed in appendix

\footnotetext{
${ }^{3}$ For the investigation of channels other than the P-wave a different $u_{0}(\mathbf{r})$ has to be considered[12].
} 
$\mathrm{C}$ of ref.[11]. The indices $n s$ and $s$ refer to non-strange and strange states, respectively. The valence quark occupation number $\eta_{\text {val }}=0,1$ is fixed such that a system with unit baryon number is described. On the other hand the bilocal kernel $\Phi^{(2)}\left(\omega ; r, r^{\prime}\right)$ receives contributions from $\mathcal{A}_{\text {val }}$ and $\mathcal{A}_{\text {vac }}$ only

$$
\begin{gathered}
\Phi^{(2)}\left(\omega ; r, r^{\prime}\right)=-\frac{N_{C}}{4}\left(m+m_{s}\right)^{2} \int \frac{d \Omega}{4 \pi} \int \frac{d \Omega^{\prime}}{4 \pi}\left\{\eta_{\text {val }} \sum_{\rho=s} \frac{\dot{\psi}_{\text {val }}^{\dagger}(\mathbf{r}) \psi_{\rho}(\mathbf{r}) \psi_{\rho}^{\dagger}\left(\mathbf{r}^{\prime}\right) \dot{\psi}_{\mathrm{val}}\left(\mathbf{r}^{\prime}\right)}{\epsilon_{\mathrm{val}}-\omega-\epsilon_{\rho}}\right. \\
\left.-\sum_{\substack{\mu=n s \\
\rho=s}} \tilde{\psi}_{\mu}^{\dagger}(\mathbf{r}) \psi_{\rho}(\mathbf{r}) \psi_{\rho}^{\dagger}\left(\mathbf{r}^{\prime}\right) \dot{\psi}_{\mu}\left(\mathbf{r}^{\prime}\right) \mathcal{R}_{\mu, \rho}(\omega)\right\} .
\end{gathered}
$$

The regulator function appearing eqn.(21) may readily be obtained from ref.[11]

$$
\begin{array}{r}
\mathcal{R}_{\mu, \rho}(\omega)=\int_{1 / \Lambda^{2}}^{\infty} d s \sqrt{\frac{s}{\pi}}\left\{\frac{\mathrm{e}^{-s \epsilon_{\mu}^{2}}+\mathrm{e}^{-s \epsilon_{\rho}^{2}}}{s}+\left[\omega^{2}-\left(\epsilon_{\mu}+\epsilon_{\rho}\right)^{2}\right] R_{0}\left(s ; \omega, \epsilon_{\mu}, \epsilon_{\rho}\right)\right. \\
\left.-2 \omega \epsilon_{\rho} R_{1}\left(s ; \omega, \epsilon_{\mu}, \epsilon_{\rho}\right)+2 \omega \epsilon_{\mu} R_{1}\left(s ; \omega, \epsilon_{\rho}, \epsilon_{\mu}\right)\right\} .
\end{array}
$$

Here $R_{i}$ denote Feynman parameter integrals

$$
R_{i}\left(s ; \omega, \epsilon_{\mu}, \epsilon_{\nu}\right)=\int_{0}^{1} x^{i} d x \exp \left(-s\left[(1-x) \epsilon_{\mu}^{2}+x \epsilon_{\nu}^{2}-x(1-x) \omega^{2}\right]\right)
$$

which need to be evaluated numerically. Varying (18) with respect to $\eta_{w}(r)$ yields the equation of motion

$$
r^{2}\left\{\int d r^{\prime} r^{\prime 2} \Phi^{(2)}\left(\omega ; r, r^{\prime}\right) \eta_{\omega}\left(r^{\prime}\right)+\Phi^{(1)}(\omega ; r) \eta_{\omega}(r)\right\}=0 .
$$

The main purpose of the program "bpwave" is to numerically solve this homogeneous linear integral equation. I.e. $\omega$ has to be adjusted such eqn.(24) is satisfied for a non-trivial function $\eta_{\omega}(r)$. This value of $\omega$ is refered to as the bound state energy and $\eta_{\omega}(r)$ as the bound state wave-function. Eqn.(24) represents the Bethe-Salpeter equation for the wave-function $\eta_{\omega}(r)$. For the numerical treatment it will be very helpful to divide the bilocal kernel (21) into several pieces according to the appearance of $\omega$ in eqn.(22)

$$
\Phi^{(2)}\left(\omega ; r, r^{\prime}\right)=\omega^{2} \Phi_{2}^{(2)}\left(\omega ; r, r^{\prime}\right)+\omega \Phi_{1}^{(2)}\left(\omega ; r, r^{\prime}\right)+\Phi_{0}^{(2)}\left(\omega ; r, r^{\prime}\right)+\Phi_{\mathrm{val}}^{(2)}\left(\omega ; r, r^{\prime}\right) .
$$

The kernels $\Phi_{i}^{(2)}(i=0,1,2)$ depend on $\omega$ only via the Feynman parameter integrals (23). $\Phi_{\text {val }}^{(2)}$ represents the term proportional to $\eta_{\text {val }}$ in (21).

Obviously eqn.(24) does not determine the normalization of the bound wave-function $\eta_{\omega}(r)$. We fix the normalization by demanding the bound state to carry strangeness charge $S= \pm 1$. The evaluation of $S$ for the bound state has been carried out in appendix A of ref.[11] and may be summarized as

$$
\begin{aligned}
S=\left(a_{1}^{\dagger}(\omega) a_{1}(\omega)\right. & \left.+a_{2}^{\dagger}(\omega) a_{2}(\omega)\right) \\
& \times \int d r r^{2}\left\{\int d r^{\prime} r^{\prime 2} \Phi_{S}^{(2)}\left(\omega ; r, r^{\prime}\right) \eta_{\omega}(r) \eta_{\omega}\left(r^{\prime}\right)+\Phi_{S}^{(1)}(r) \eta_{\omega}(r) \eta_{\omega}(r)\right\}
\end{aligned}
$$


with

$$
\begin{aligned}
\Phi_{S}^{(1)}(r)=-\frac{N_{C}}{4}\left(m+m_{s}\right) & \int_{1 / \Lambda^{2}}^{\infty} \frac{d s}{\sqrt{4 \pi s}} \int \frac{d \Omega}{4 \pi} \sum_{\rho=s} e^{-s \epsilon_{\rho}^{2}} \\
& \times \psi_{\rho}^{\dagger}(\mathbf{r})\left\{\left(1-2 s \epsilon_{\rho}^{2}\right) \beta+s \epsilon_{\rho}\left(m+m_{s}\right)\right\} \psi_{\rho}(\mathbf{r})
\end{aligned}
$$

and

$$
\begin{aligned}
& \Phi_{S}^{(2)}\left(\omega ; r, r^{\prime}\right)=-\frac{N_{C}}{4}\left(m+m_{s}\right)^{2} \int \frac{d \Omega}{4 \pi} \int \frac{d \Omega^{\prime}}{4 \pi}\left\{\eta_{\mathrm{val}} \sum_{\rho=s} \frac{\psi_{\mathrm{val}}^{\dagger}(\mathbf{r}) \psi_{\rho}(\mathbf{r}) \psi_{\rho}^{\dagger}\left(\mathbf{r}^{\prime}\right) \dot{\psi}_{\mathrm{val}}\left(\mathbf{r}^{\prime}\right)}{\left(\epsilon_{\mathrm{val}}-\omega-\epsilon_{\rho}\right)^{2}}\right. \\
& \left.-\sum_{\substack{\mu=n s \\
\rho=s}} \tilde{\psi}_{\mu}^{\dagger}(\mathbf{r}) \psi_{\rho}(\mathbf{r}) \psi_{\rho}^{\dagger}\left(\mathbf{r}^{\prime}\right) \dot{\psi}_{\mu}\left(\mathbf{r}^{\prime}\right) \mathcal{R}_{\mu, \rho}^{S}(\omega)\right\} \text {. }
\end{aligned}
$$

The regulator function in (28) is given by[11]

$$
\begin{aligned}
\mathcal{R}_{\mu, \rho}^{S}(\omega)=\left(\omega-\epsilon_{\mu}-\epsilon_{\rho}\right) & \int_{1 / \Lambda^{2}}^{\infty} d s \sqrt{\frac{s}{4 \pi}}\left\{R_{0}\left(s ; \omega, \epsilon_{\mu}, \epsilon_{\rho}\right)\right. \\
& \left.-s\left(\omega+\epsilon_{\mu}+\epsilon_{\rho}\right)\left(\left(\epsilon_{\rho}-\omega\right) R_{1}\left(s ; \omega, \epsilon_{\mu}, \epsilon_{\rho}\right)+\omega R_{2}\left(s ; \omega, \epsilon_{\mu}, \epsilon_{\rho}\right)\right)\right\} .
\end{aligned}
$$

The normalization condition finally reads

$$
\left|\int d r r^{2}\left\{\int d r^{\prime} r^{\prime 2} \Phi_{S}^{(2)}\left(\omega ; r, r^{\prime}\right) \eta_{\omega}(r) \eta_{\omega}\left(r^{\prime}\right)+\Phi_{S}^{(1)}(r) \eta_{\omega}(r) \eta_{\omega}(r)\right\}\right|=1
$$

which together with the Bethe-Salpeter equation (24) completely determines the bound state wave-function $\eta_{\omega}(r)$ up to an irrelevant sign.

\section{Numerical methods}

\subsection{Eigenstates of the static Hamiltonian}

The eigenvalues and -states of the static Hamiltonian (12,13) are required. Since $h_{(0)}(13)$ commutes with the grand spin operator $\mathbf{G}(14)$ the eigenstates are constructed such that they diagonalize $h_{(0)}, \mathrm{G}^{2}$ and $\mathrm{G}_{3}$. For the free Hamiltonian (i.e. $\Theta=0$ in eqn.(13)) the eigenstates in the non-strange sector are

$$
\begin{aligned}
\left|1, n, j=G+\frac{1}{2}, M\right\rangle & =\mathcal{N}_{n}^{G}\left(\begin{array}{c}
i w_{n G}^{+} j_{G}\left(k_{n G} r\right)\left|G G+\frac{1}{2} G M\right\rangle \\
w_{n G}^{-} j_{G+1}\left(k_{n G} r\right)\left|G+1 G+\frac{1}{2} G M\right\rangle
\end{array}\right), \\
\epsilon^{0} & =E_{n G}= \pm \sqrt{k_{n G}^{2}+m^{2}}, \quad \Pi=(-)^{G} . \\
\left|2, n, j=G-\frac{1}{2}, M\right\rangle & =\mathcal{V}_{n}^{G}\left(\begin{array}{c}
i w_{n G}^{+} j_{G}\left(k_{n G} r\right)\left|G G-\frac{1}{2} G M\right\rangle \\
-w_{n G}^{-} j_{G-1}\left(k_{n G} r\right)\left|G-1 G-\frac{1}{2} G M\right\rangle
\end{array}\right), \\
\epsilon^{0} & =E_{n G}= \pm \sqrt{k_{n G}^{2}+m^{2}}, \quad \Pi=(-)^{G} .
\end{aligned}
$$




$$
\begin{aligned}
\left|3, n, j=G+\frac{1}{2}, M\right\rangle & =\mathcal{N}_{n}^{G+1}\left(\begin{array}{c}
i w_{n G+1}^{+} j_{G+1}\left(k_{n G+1} r\right)\left|G+1 G+\frac{1}{2} G M\right\rangle \\
-w_{n G+1}^{-} j_{G}\left(k_{n G+1} r\right)\left|G G+\frac{1}{2} G M\right\rangle
\end{array}\right), \\
e^{0} & =E_{n G+1}= \pm \sqrt{k_{n G+1}^{2}+m^{2}}, \quad \Pi=(-)^{G+1} \\
\left|4, n, j=G-\frac{1}{2}, M\right\rangle & =\mathcal{N}_{n}^{G-1}\left(\begin{array}{c}
i w_{n G-1}^{+} j_{G-1}\left(k_{n G-1} r\right)\left|G-1 G-\frac{1}{2} G M\right\rangle \\
w_{n G-1}^{-} j_{G}\left(k_{n G-1} r\right)\left|G G-\frac{1}{2} G M\right\rangle
\end{array}\right), \\
\epsilon^{0} & =E_{n G-1}= \pm \sqrt{k_{n G-1}^{2}+m^{2}}, \quad \Pi=(-)^{G+1}
\end{aligned}
$$

wherein $j_{l}$ denote the spherical Bessel functions. Also the energy eigenvalues $\epsilon^{0}$ of the free Hamiltonian and the parity quantum numbers $\Pi$ are listed. $|l j G M\rangle$ represent states where first the orbital angular momentum $l$ and the spin are coupled to the total angular moment um $j=l \pm 1 / 2$ and secondly isospin and $j$ are coupled to the grand spin $G . M$ is the projection of $G$. This coupling scheme leads to the selection rules

$$
j= \begin{cases}G+1 / 2, & l=\left\{\begin{array}{l}
G+1 \\
G
\end{array}\right. \\
G-1 / 2, & l=\left\{\begin{array}{l}
G \\
G-1
\end{array} .\right.\end{cases}
$$

The kinematical factors $w_{n l}^{ \pm}$are defined as

$$
w_{n l}^{+}=\sqrt{1+m / E_{n l}}, \quad w_{n l}^{-}=\operatorname{sign}\left(E_{n l}\right) \sqrt{1-m / E_{n l}} .
$$

This system is treated in a spherical box of finite radius $D$. Thereby the momentum eigenvalues are discretized by demanding the upper components to vanish at the boundary, i.e.

$$
j_{l}\left(k_{n l} D\right)=0 .
$$

The normalization is given by

$$
\mathcal{N}_{n}^{l}=\left[D^{3 / 2}\left|j_{l+1}\left(k_{n l} D\right)\right|\right]^{-1} .
$$

The numerical diagonalization of $h_{(0)}$ requires an upper bound, $k_{\max }$ for the momenta $k_{n l}$ leaving for each $l$ a specified number of momentum eigenstates $N(l): k_{1 l}, \ldots, k_{N(l) !}[5]$. Taking into account that for each momentum one has two energy eigenvalues $\pm e^{0}$ it is obvious that we have $2 N(l)$ eigenfunctions of $h_{0}(\Theta=0)$ for each grand spinor in eqn.(31).

The corresponding eigenstates of the zeroth-order Hamiltonian $h_{(0)}$ are linear combinations of the spinors in (31). The eigenstates with parity $\Pi=(-)^{G}$ read:

$$
\begin{aligned}
|\mu, G, M,+\rangle= & \sum_{n=1}^{2 N(G)} V_{G}^{(+)}(n, \mu)|1, n, G, M\rangle \\
& +\sum_{n=1}^{2 N(G)} V_{G}^{(+)}(n+2 N(G), \mu)|2, n, G, M\rangle
\end{aligned}
$$

while those with parity $\Pi=(-)^{G+1}$ are given by

$$
\begin{aligned}
|\mu, G, M,-\rangle= & \sum_{n=1}^{2 N(G+1)} V_{G}^{(-)}(n, \mu)|3, n, G, M\rangle \\
& +\sum_{n=1}^{2 N(G-1)} V_{G}^{(-)}(n+2 N(G+1), \mu)|4, n, G, M\rangle .
\end{aligned}
$$


The eigenvectors $V_{G}^{ \pm}(n, \mu)$ and the corresponding eigenvalues $\epsilon_{\mu}$ of $h_{(0)}$ are calculated using the numerical routine "chisol" [5]. This routine stores $V_{G}^{ \pm}(n, \mu)$ and $\epsilon_{\mu}$ together with the free energies $E_{n G}$ and momenta $k_{n G}$ in the unformatted file "VEC.DAT" which serves as input file for the current program. The eigenvectors $V_{G}^{ \pm}(n, \mu)$ are used to construct the coordinate space representation of the eigenstates $|\mu\rangle$ for positive and negative intrinsic parity

$$
\begin{aligned}
& \Psi_{\mu}^{(G,+)}=\left(\begin{array}{c}
i g_{\mu}^{(G,+; 1)}(r)\left|G G+\frac{1}{2} G M\right\rangle \\
f_{\mu}^{(G,+1)}(r)\left|G+1 G+\frac{1}{2} G M\right\rangle
\end{array}\right)+\left(\begin{array}{c}
i g_{\mu}^{(G,+; 2)}(r)\left|G G-\frac{1}{2} G M\right\rangle \\
-f_{\mu}^{(G,+; 2)}(r)\left|G-1 G-\frac{1}{2} G M\right\rangle
\end{array}\right) \\
& \Psi_{\mu}^{(G,-)}=\left(\begin{array}{c}
i g_{\mu}^{(G,-; 1)}(r)\left|G+1 G+\frac{1}{2} G M\right\rangle \\
-f_{\mu}^{(G,-; 1)}(r)\left|G G+\frac{1}{2} G M\right\rangle
\end{array}\right)+\left(\begin{array}{c}
i g_{\mu}^{(G,-; 2)}(r)\left|G-1 G-\frac{1}{2} G M\right\rangle \\
f_{\mu}^{(G,-; 2)}(r)\left|G G-\frac{1}{2} G M\right\rangle
\end{array}\right) .
\end{aligned}
$$

The radial functions $g_{\mu}^{(G,+; 1)}(r), f_{\mu}^{(G,+; 1)}(r)$, etc. involve the eigenvectors, $\boldsymbol{\epsilon} . g$.

$$
g_{\mu}^{(G,+; 1)}(r)=\sum_{n=1}^{2 N(G)} V_{G}^{(+)}(n, \mu) \mathcal{N}_{n}^{G} w_{n G}^{+} j_{G}\left(k_{n G} r\right) .
$$

Next the unitary transformation (19) defines new radial functions $\dot{g}_{\mu}^{G,+; 1}(r)$, etc. via

$$
\begin{gathered}
\dot{\Psi}_{\mu}^{(G,+)}=\left(\begin{array}{c}
i \dot{g}_{\mu}^{(G,+; 1)}(r)\left|G G+\frac{1}{2} G M\right\rangle \\
\tilde{f}_{\mu}^{(G,+; 1)}(r)\left|G+1 G+\frac{1}{2} G M\right\rangle
\end{array}\right)+\left(\begin{array}{c}
i \dot{g}_{\mu}^{(G,+; 2)}(r)\left|G G-\frac{1}{2} G M\right\rangle \\
-\dot{f}_{\mu}^{(G,+; 2)}(r)\left|G-1 G-\frac{1}{2} G M\right\rangle
\end{array}\right), \\
\dot{\Psi}_{\mu}^{(G,-)}=\left(\begin{array}{c}
i \hat{g}_{\mu}^{(G,-; 1)}(r)\left|G+1 G+\frac{1}{2} G M\right\rangle \\
-\tilde{f}_{\mu}^{(G,-; 1)}(r)\left|G G+\frac{1}{2} G M\right\rangle
\end{array}\right)+\left(\begin{array}{c}
i \dot{g}_{\mu}^{(G,-; 2)}(r)\left|G-1 G-\frac{1}{2} G M\right\rangle \\
\tilde{f}_{\mu}^{(G,-; 2)}(r)\left|G G-\frac{1}{2} G M\right\rangle
\end{array}\right) .
\end{gathered}
$$

As an example we display

$$
\dot{g}_{\mu}^{(G,+; 1)}(r)=\sin \frac{\Theta}{2} g_{\mu}^{(G,+; 1)}(r)-\frac{1}{2 G+1} \cos \frac{\Theta}{2} f_{\mu}^{(G,+; 1)}(r)-\frac{2 \sqrt{G(G+1)}}{2 G+1} \cos \frac{\Theta}{2} f_{\mu}^{(G,+; 2)}(r) .
$$

The strange quarks have vanishing isospin. Thus their grand spin is identical to their ordinary spin. We classify the basis spinors by their total spin $j$ and orbital angular momentum $l$ :

$$
\begin{aligned}
\left|1, \rho, j=l+\frac{1}{2}, m\right\rangle_{s} & =\mathcal{N}_{\rho}^{l}\left(\begin{array}{c}
i \bar{w}_{\rho l}^{+} j_{l}\left(k_{\rho l} r\right)|l j m\rangle \\
\bar{w}_{\rho l}^{-} j_{l+1}\left(k_{\rho l} r\right)|l+1 j m\rangle
\end{array}\right), \\
\epsilon^{0} & =\bar{E}_{\rho l}= \pm \sqrt{k_{\rho l}^{2}+m_{s}^{2}} . \\
\left|2, \rho, j=l-\frac{1}{2}, m\right\rangle_{s} & =\mathcal{N}_{\rho}^{l}\left(\begin{array}{c}
i \bar{w}_{\rho l}^{+} j_{l}\left(k_{\rho l} r\right)|l j m\rangle \\
-\bar{w}_{\rho l}^{-} j_{l-1}\left(k_{\rho l} r\right)|l-1 j m\rangle
\end{array}\right), \\
\epsilon^{0} & =\bar{E}_{\rho l}= \pm \sqrt{k_{\rho l}^{2}+m_{s}^{2}} . \\
\bar{w}_{\rho l}^{+}=\sqrt{1+m_{s} / \bar{E}_{\rho l}}, & \bar{w}_{\rho l}^{-}=\operatorname{sign}\left(\bar{E}_{\rho l}\right) \sqrt{1-m_{s} / \bar{E}_{\rho l} .}
\end{aligned}
$$

Note the appearance of the strange constituent mass $m_{s}$ in the energy eigenvalues. Since the chiral soliton has vanishing strangeness the eigenstates (42) of the free Hamiltonian are also eigenstates of the zeroth-order Hamiltonian (13). 


\subsection{The Bethe-Salpeter equation}

Now we have constructed all wave-functions appearing in the kernels $\Phi^{(1)}, \Phi^{(2)}, \Phi_{S}^{(1)}$ and $\Phi_{S}^{(2)}$. Thus we are enabled to calculated their radial dependencies. However, we still have to evaluate the regulator functions appearing in eqns.(20,21,27 and 28$)$. These are of the general structure

$$
\int_{1 / \Lambda^{2}}^{\infty} d s s^{\frac{n}{2}} \exp (-s A), \quad n=-1,1,3 .
$$

Here $A$ is a function of the frequency $\omega$ as well as of the eigenvalues $\epsilon_{\mu}$ (12). It should be remarked that for our purposes $A$ is always positive. Integration by parts

$$
\int_{1 / \Lambda^{2}}^{\infty} d s s^{\frac{n}{2}} \exp (-s A)=-\frac{1}{A} \Lambda^{-n} \exp \left(-\frac{A}{\Lambda^{2}}\right)+\frac{n}{2 A} \int_{1 / \Lambda^{2}}^{\infty} d s s^{\frac{n}{2}-1} \exp (-s A)
$$

allows to relate the regulator functions to the complementary error function

$$
\int_{1 / \Lambda^{2}}^{\infty} \frac{d s}{\sqrt{\pi s}} \exp (-s A)=\frac{1}{|A|} \operatorname{erfc}\left(\left|\frac{A}{\Lambda}\right|\right)
$$

which can be obtained numerically[13]. This finally enables us to evaluate the kernels for a given frequency $\omega$ as mode sums involving the wave-functions defined above.

By symmetry arguments it can be shown that $\Phi_{1}^{(2)}\left(\omega ; r, r^{\prime}\right)(25)$ vanishes for $\Theta=0$. For the boundary conditions (34) this is only achieved in the continuum limit $D \rightarrow \infty$. Thus for finite $D$ the corresponding result needs to be subtracted.

For numerical calculations the radial coordinate is discretized $r_{i}=i \Delta r$ with $i=0, \ldots, N$, providing matrices $\Phi_{i j}^{(2)}(\omega)=\Phi^{(2)}\left(\omega ; r_{i}, r_{j}\right)$, etc.. Then the Bethe-Salpeter equation becomes a matrix equation

$$
r_{i}^{2} \sum_{j=0}^{N}\left\{\Delta r r_{j}^{2} \Phi_{i j}^{(2)}(\omega)+\delta_{i j} \Phi_{j}^{(1)}(\omega)\right\} \eta_{j}(\omega)=0 \quad i=0, \ldots, N .
$$

For $i=0$ this equation is trivially satisfied since the kernels are regular. Therefore this point has to be treated separately. For $i, j \neq 0$ we extend (47) to an eigenvalue problem

$$
r_{i}^{2} \sum_{j>0}\left\{\Delta r r_{j}^{2} \Phi_{i j}^{(2)}(\omega)+\delta_{i j} \Phi_{j}^{(1)}\right\} \eta_{j}(\omega)=\lambda(\omega) \eta_{i}(\omega) .
$$

The symmetric coefficient matrix on the LHS possesses real eigenvalues $\lambda$ which are computed for a given frequency $\omega$. Finally $\omega$ is tuned to $\omega_{0}$ such that (48) has a solution with the eigenvalue $\lambda\left(\omega_{0}\right)=0$.

It is quite tricky to find this solution without abusing lots of computational time. In this context it is important to note that the evaluation of the Feynman parameter integrals (23) consumes most of the computational time. Thus the application of e.g. a bisection algorithm to solve (48) for $\lambda\left(\omega_{0}\right)=0$ would be very slow. We therefore try to find this solution in two steps making use of the fact that the Feynman parameter integrals vary only slowly with $\omega$. For a first guess, $\omega_{G}$ we calculate the bilocal kernels $(25) \Phi_{k i j}^{(2)}\left(\omega_{G}\right)=\Phi_{k}^{(2)}\left(\omega_{G} ; r_{i}, r_{j}\right)$, $k=0,1,2$ as well as the local kernel $\Phi_{j}^{(1)}$. It is then easy to numerically adjust $\omega_{P}$ such that

$$
\operatorname{det}\left\{r_{i}^{2} r_{j}^{2} \triangle r\left[\omega_{P}^{2} \Phi_{2 i j}^{(2)}\left(\omega_{G}\right)+\omega_{P} \Phi_{1}^{(2)}\left(\omega_{G}\right)+\Phi_{0}^{(2)}\left(\omega_{G}\right)+\Phi_{\text {val } i j}^{(2)}\left(\omega_{P}\right)\right]+\delta_{i j} \Phi_{i}^{(1)} r_{i}^{2}\right\}=0
$$


Then we set $\omega_{G}=\omega_{P}$ and re-evaluate the bilocal kernels $\Phi_{k i j}^{(2)}\left(\omega_{G}\right)$. For the new kernels again a solution to (49) is constructed. This procedure is iterated until the deviation of the solution to (49) from the argument of the kernels falls short of a certain value. Since the kernels $\Phi_{k i j}^{(2)}(\omega)$ vary only slowly with $\omega$ this happens already after a few interations ${ }^{4}$. Then the solution to the Bethe-Salpeter (48) equation is given by $\omega_{P}=\omega_{G}=: \omega_{0}$. It also yields the eigenvector $\eta_{i}\left(\omega_{0}\right) i>0$.

Having obtained the solution to (48) we may also evaluate the wave-function at the origin $(i=0)$

$$
\eta_{0}\left(\omega_{0}\right)=-\frac{1}{\Phi_{0}^{(1)}\left(\omega_{0}\right)} \sum_{j>0} \Delta r r_{j}^{2} \Phi_{0 j}^{(2)}\left(\omega_{0}\right) \eta_{j}\left(\omega_{0}\right)
$$

since neither $\Phi^{(2)}\left(\omega ; r, r^{\prime}\right)$ nor $\Phi^{(1)}(\omega ; r)$ vanish at the origin. The resulting eigenvector $\eta_{i}(\omega)$ is identified as the discretized solution of eqn.(24). Finally the discretized version of (30) is employed to normalize $\eta_{i}\left(\omega_{0}\right)$.

\section{Description of the program}

\subsection{The main program}

The purpose of the main program is to carry out the mode sums involved in the kernels (21) (see also eqns.(25) and (49)) for a given $\omega=\omega_{G}$ and construct the solution to the Bethe-Salpeter equation (24). The evaluation of the regulator functions is performed in subroutines.

The routine starts by reading the self-consistent soliton profile from the file "chi.in" which is created by the routine "chisol" [5]. From this file also the empirical values for $m_{\pi}, f_{\pi}$ and $m_{k}$ as well as the only undetermined parameter $m$ are taken as input quantities. According to eqns. $(3-8)$ the parameters of the N.JL model with proper time regularization $(e . g . \quad \Lambda)$ are calculated. Next the lattice $r_{i}=i \Delta r$ is defined and the soliton profile is interpolated to these lattice points using a Laguerre algorithm.

The mode sums are organized as follows. The contributions from different grand spin $(G)$ channels are evaluated subsequently. Each grand spin channel splits into positive and negative intrinsic parity parts $(\pi= \pm)$. In this way the channels $G^{\pi}$ are defined. The program reads the eigenvectors $V_{G}^{ \pm}(n, \mu)$, eigenvalues $\epsilon_{\mu}$ and momenta $k_{n G}$ from the file "VEC.DAT" (also created by the routine "chisol"[5]). From this the kinematical quantities like $w_{n 1}(33,43)$ and the radial functions $\grave{g}_{\mu}^{(G,+; 1)}$, etc. (41) of the transformed wave functions are constructed for each channel $G^{\pi}$. In the $0^{+}$channel the valence quark state is identified. For non-strange states the mode sum corresponds to the sum over the states $|\mu\rangle$ for which the coordinate space representation of the wave-function is now at hand while for the (free) strange states one just sums over momentum eigenstates. The storage of the contribution from these states at distinct lattice points is left as the innermost loop. This structure has a very important reason. As already mentioned the most computing time consuming part of the program is represented by the evaluation of the Feynman parameter integrals (23). It is therefore necessary to carry out these integrals as seldom as possible. Although this structure reduces the computation time it has the disadvantage that the radial functions $\dot{g}_{\mu}^{G,+; 1}(r)(41)$ are

\footnotetext{
${ }^{4}$ The number of iterations obviously depends on the desired accuracy.
} 
stored in an array. For this storage memory space at the amount of a few MB is needed. A Gauß-Legendre algorithm is used to calculate the Feynman parameter integrals. It turns out that a high precision can already be obtained with only a few abscissas. However, there is one exception. In case one of the energy eigenvalues $\epsilon_{\mu}$ or $\epsilon_{\nu}$ in (23) is close to zero a high density of abscissas is needed. Fortunately there is only one such eigenvalue of $h_{(0)}: \epsilon_{\mathrm{val}}$. The program provides two sets of abscissas and weights for the Gauß-Legendre algorithm and only when $\epsilon_{\text {val }}$ is involved the denser set is employed. The sum over grand spins is terminated when ail eigenstates of the free Hamiltonian $h_{(0)}(\Theta=0)$ carry momenta larger then the upper bound $k_{\max }$. Subtracting the result of the trivial configuration $(\Theta=0)$ for $\Phi_{1}^{(2)}(25)$ completes the calculation of the vacuum contribution to the kernels. Then we employ a bisection algorithm to solve (49) in a given interval $\omega_{G}-\delta \leq \omega_{P} \leq \omega_{G}+\delta$. It should be noted that the full $\omega_{P}$ dependence of the valence quark $\Phi_{\text {val }}^{(2)}$ is always kept when looking for the solution $\omega_{P}$ to (49). In case the valence quark has joined the Dirac sea $\left(\epsilon_{\mathrm{val}}<0\right) \Phi_{\text {val }}^{(2)}$ must not be included.

Then this solution $\omega_{P}$ is taken as an improved guess $\omega_{G}$ for which the kernels $\Phi_{k}^{(2)}\left(\omega_{G}\right)$ are re-evaluated. These kernels are again inserted into (49) and a new solution $\omega_{P}$ is obtained. This procedure is repeated until the condition $\left|\omega_{P}-\omega_{G}\right| \leq \delta^{\prime}$ is fulfilled.

Finally the associated wave-function is constructed and normalized according to (30).

\subsection{Subroutines and functions}

Function ylagn

Performs the Laguerre interpolation of a given function.

Subroutine point

Provides points and weights for the Laguerre interpolation.

Function $y$

Calculates Bessel functions $j_{l}(z)$.

Function erfcc

Calculates the complementary error function erfc(x). Cf. chapter 6.2 in ref.[13].

Function frr

Calculates the regulator function for the local kernel $\Phi^{(1)}(r)$.

Function gar0

Calculates the regulator function for the bilocal kernel $\Phi_{0}^{(2)}\left(\omega ; r, r^{\prime}\right)$.

Function gar 1

Calculates the regulator function for the bilocal kernel $\Phi_{1}^{(2)}\left(\omega ; r, r^{\prime}\right)$.

Function gar10

Calculates of the regulator function needed for the subtraction in $\Phi_{1}^{(2)}$ when $\Theta=0$.

Function gare

Calculates the regulator function for the bilocal kernel $\Phi_{2}^{(2)}\left(\omega ; r, r^{\prime}\right)$.

Function $f s 1$

Calculates the regulator function for the local kernel $\Phi_{S}^{(1)}(r)$.

Function gas 2

Calculates the regulator function for the bilocal kernel $\Phi_{S}^{(2)}\left(\omega ; r, r^{\prime}\right)$.

Subroutine gauleg

Provides points and weights for the Gauß-Legendre integration. Cf. chapter 4.5 in ref.[13]. Subroutine jacobi

Diagonalizes a symmetric matrix. Cf. chapter 11.1 in ref.[13]. 
Function bode

Calculates radial integrals.

Function $g$

Calculates the kernel of the Bethe-Salpeter equation for free pions.

Function $d g$

Derivative of $g$ with respect to $(m / \Lambda)^{2}$.

Function gamma0

Calculates the incomplete $\Gamma$-function $\Gamma(0, x)$.

Function $f k k$

Calculates the kaon decay constant $f_{K}$.

Function ypol

Provides the kernel of the Bethe-Salpeter equation for free kaons.

Function rtbis

Solves the Bethe-Salpeter equation for free kaons using a bisection method.

\section{Testing the program}

Trivial tests establishing the independence of the lattice size $\Delta r$, the box radius $D$ or the upper bound for the momentum states $k_{\max }$ have, of course, been performed.

Fortunately, there are two further tests at hand. These are physically motivated and deal with (i) kaon fields for a trivial background configuration $(\Theta=0)$ in a spherical box and (ii) the SU(3) symmetric model $\left(m^{0}=m_{s}^{0}\right)$.

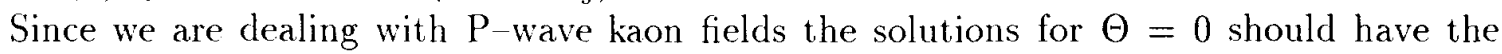
shape $j_{1}\left(k_{n 1} r\right)$. As these configurations are not localized the treatment in a spherical box of finite radius $D$ may not be the most appropriate, especially the discretized Bethe-Salpeter equation (47) allows only for solutions which vanish at $r=D$. The corresponding "bound state" energies are $\sqrt{M_{S}^{2}+k_{n 1}^{2}}$. Of course, this is nothing but the dispersion relation and should yield the kaon mass $M_{S}=m_{K}$. Our solutions to the discretized Bethe-Salpeter equation approximately reproduce the functional behavior of the first Bessel function and $M_{S}$ is found to be about $501 \mathrm{MeV}$ i.e. the numerical error is of the order $1 \%$. To obtain this result we have used $D=5 \mathrm{fm}$ and $\triangle r=0.005 D$. Further increase of $D$ and/or decrease of $\Delta r$ reduce the above mentioned error.

In the SU(3) symmetric limit the theory contains a zero-mode corresponding to an infinitesimal rotation of the static solution into $\mathrm{SU}(3)$ direction. Analytically it can be shown that it corresponds to $\eta_{0}(r)=$ Const. $\times \sin \Theta / 2$. Substituting $m_{s}=m$ and $m_{s}^{0}=m^{0}$ the program reproduces this zero mode at $\omega \approx-0.15 \mathrm{MeV}$. Taking into account that the energy scale involved is $m$ (which is of the order of several hundred MeV) this check comes out perfectly well. Also the deviation of the corresponding numerical wave-function from Const. $\times \sin \Theta / 2$ is negligible. This provides clear evidence that the program is well suited for the evaluation of localized bound states.

\section{Acknowledgements}

We gratefully acknowledge the valuable collaboration with $\mathrm{H}$. Reinhardt. HW would also like to thank B. Schwesinger for contributing on the subroutines ylagn and points. 


\section{References}

[1] H. Reinhardt and R. Wünsch, Phys. Lett. B215 (1988) 577; Phys. Lett. B230 (1989) 93 ;

T. Meissner, F. Grümmer and K. Goeke, Phys. Lett. B227 (1989) 296;

R. Alkofer, Phys. Lett. B236 (1990) 310.

[2] Y. Nambu and G. Jona-Lasinio, Phys. Rev. 122 (1961) 345; 124 (1961) 246.

[3] C. Callan and I. Klebanov, Nucl. Phys. B262 (1985) 365;

C. Callan, K. Hornbostel and I. Klebanov, Phys. Lett. B202 (1988) 296;

I. Klebanov in Hadrons and Hadronic Matter, page 223, proceedings of the NATO Advanced Study Institute, Cargese, 1989, edited by D. Vautherin, J. Negele and F. Lenz (Plenum Press 1989).

[4] T. H. R. Skyrme, Proc. Roy. Soc. A260 (1961) 127;

G. S. Adkins, C. R. Nappi and E. Witten, Nucl. Phys. B228 (1983) 552;

see also "Baryons as Skyrme Solitons", G. Holzwarth (Ed.), World Scientific Publ. Comp., Singapore 1993.

[5] R. Alkofer and H. Weigel, "Self-consistent solution to a fermion determinant with space dependent fields", UNITU-THEP-1/1994. Submitted to Computer Physics Communications.

[6] D. Ebert and H. Reinhardt, Nucl. Phys. B271 (1986) 188.

[7] J. Schwinger, Phys. Rev. 82 (1951) 664.

[8] H. Weigel, R. Alkofer and H. Reinhardt, Nucl. Phys. B387 (1992) 638.

[9] H. Weigel, H. Reinhardt and R. Alkofer, Phys. Lett. B313 (1993) 377.

[10] H. Reinhardt, Nucl. Phys. A503 (1989) 825.

[11] H. Weigel, R. Alkofer and H. Reinhardt, "Hyperons in the bound state approach to the Nambu--Jona-Lasinio chiral soliton", Tübingen preprint Oct. 93, HEP-PH/9310309.

[12] H. Weigel, R. Alkofer and H. Reinhardt, "Strange S-Wave Excitation of the NJL Soliton" Tübingen preprint Dec. 93, HEP-PH/9312269. Phys. Rev. D, in press.

[13] W. A. Press, B. P. Flannery, S. A. Teukolsky and W. T. Vetterling, "Numerical Recipes", Cambridge University Press 1989. 
